research aims to see if small joint collateral ligament abnormalities are worth exploring further in relationship to pain in HOA.

Methods: High-resolution contrast enhanced MRI of 26 joints in 15 patients (mean (SD) age 58.3 (8.2), 13F:2M) and 10 in 5 healthy controls (age 38 (5.6); $4 \mathrm{~F}: 1 \mathrm{M})$ were scanned using a microscopy MRI coil. 15 joints in 8 patients were painful [median (IQR) pain VAS $4(3,7)$ ). Joints were scored blinded to clinical data for joint fluid, capsule/synovitis, extracapsular oedema, collateral ligament thickening/oedema/degeneration, extensor and flexor tendons, bone oedema and cysts. All structures were graded $0-3$ for normal, mild, moderate, severe, as defined in OMERACT HOAMRIS where available [2]. Proportions of joints with any level of abnormality (score $>0$ ) were calculated according to pain status (present/absent).

Results: All OA patients with and without pain had ligament abnormalities. Substantive differences in proportion of joints between healthy controls and OA patients were seen for all pathologies except tendons (no tendon abnormalities were found in all groups). Proportions of joints with capsular/synovium, extra capsular changes and proximal cysts differed between OA joints with and without pain but no substantive differences in pathology score were found. Of painful joints, 93\% (14) had both ligament and capsular/synovium or extracapsular abnormality present, compared to $45 \%$ (5) of non-painful joints.

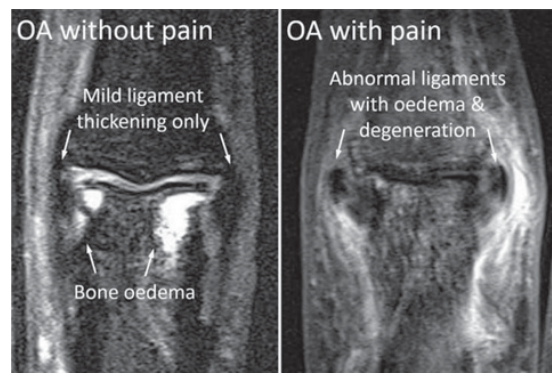

Conclusions: Modifiable abnormalities involving capsular/synovium and extracapsular areas may be more frequently seen in painful OA joints. The presence of collateral ligament abnormalities in HOA joints, whether painful or not, suggest that the severity of ligament abnormalities in small joint OA and the degree of pain may be an important area to investigate further.

References:

[1] Tan AL, et al. Ann Rheum Dis. 2006 Oct:65(10):1267-72.

[2] Haugen IK, et al. J Rheumatol. 2014 Feb;41(2):386-91.

Acknowledgements: Funded by NIHR

Disclosure of Interest: None declared

DOI: 10.1136/annrheumdis-2017-eular.3532

\section{OP0342 ASSESSMENT OF STRUCTURAL DAMAGE OF THE THUMB BASE IN PATIENTS WITH HAND OSTEOARTHRITIS: COMPARING THE NEWLY DEVELOPED OMERACT MAGNETIC RESONANCE IMAGING SCORING SYSTEM WITH STANDARD RADIOGRAPHY}

S. van Beest ${ }^{1}$, F.P. Kroon ${ }^{1}$, W. Damman ${ }^{1}$, R. Liu ${ }^{1}$, M. Kloppenburg ${ }^{1,2}$. ${ }^{1}$ Rheumatology; ${ }^{2}$ Clinical Epidemiology, Leiden University Medical Center, Leiden, Netherlands

Background: The thumb base is frequently involved in patients with hand osteoarthritis (OA), resulting in osteophytes and cartilage loss. Radiography is the most commonly used imaging modality to evaluate structural OA signs, however it is insensitive especially due to overprojection. Magnetic resonance imaging (MRI) could be a valuable alternative, however a standardized scoring method for thumb base MR images did not exist until recently OMERACT developed the thumb base OA MRI scoring system (TOMS) ${ }^{1}$

Objectives: Our aim was to investigate the validity of the new TOMS by comparing TOMS scores with radiographic scores.

Methods: Two hundred consecutively included patients $(83.5 \%$ women, median age 60.5 years) diagnosed with primary hand OA in secondary care, who had both a dorsopalmar radiograph of the right hand and a MRI scan of the right thumb base taken at baseline, were studied. T1- and fat suppressed T2-turbo spin weighted sequences were performed in axial and coronal planes on a 1.5 Tesla extremity MRI unit. Radiographs of the first carpometacarpal (CMC1) and scaphotrapeziotrapezoid (STT) joints were scored using the OARSI atlas (osteophytes and joint space narrowing [JSN] in CMC1: 0-3 and STT: absent/present) by one reader with good intra-reader reliability, blinded for clinical and MRI data. MR images were scored using TOMS (osteophytes in CMC1: 0-6 and STT: 0-9; cartilage space loss [CSL] for both joints: $0-3$ ) by two readers, blinded for clinical and radiographic data, with good intra- and inter-reader reliability. For further analysis we used the average of both readers, rounded down to the nearest integer. To study validity, the distribution of the TOMS scores for osteophytes and CSL were compared for the different radiographic stages for osteophytes and JSN, respectively.

Results: On MR images osteophytes were detected in the vast majority of thumb bases (CMC1 $n=172$; STT $n=102)$. The score of TOMS increased with more severe radiographic stages (see figures). However, the number of patients without any osteophytes in both CMC1 and STT was considerably lower for TOMS $(n=19)$ than for the OARSI $(n=106)$ scoring. A similar difference was apparent for absence of CSL $(n=82)$ versus JSN $(n=108)$ in both CMC1 and STT. Patients with isolated STT osteophytes were quite rare for both TOMS $(n=9)$ and the OARSI $(n=5)$ scoring. The most prominent discrepancy between TOMS and OARSI sensitivity was found for osteophytes: an additional 168 joints $(C M C 1 n=84 ;$ STT $n=84)$ were found positive with TOMS, while only 1 OARSI-positive CMC1 scored negative with TOMS.
Osteophytes
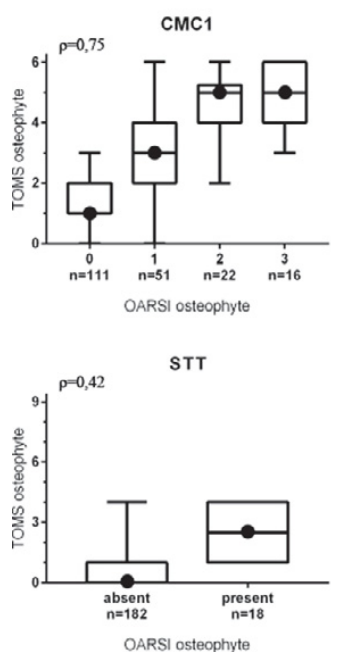

Joint space

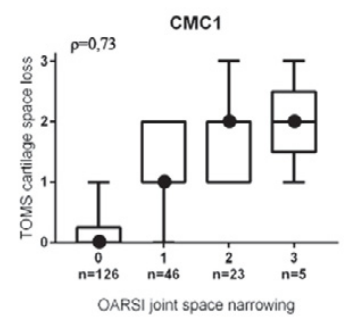

STT

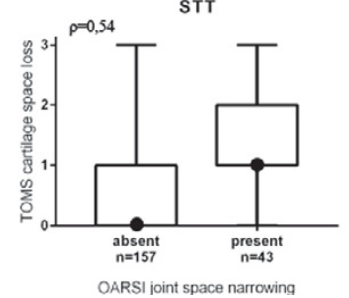

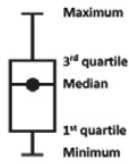

$\rho=\stackrel{\text { Spearman's ran }}{\text { correlation }}$
Conclusions: Scores of osteophytes and cartilage loss assessed on MR images by TOMS were correlated with radiographic scores, indicating good validity of the TOMS. Furthermore, the frequencies of joints with osteophytes and cartilage loss assessed on MR images were higher compared to those on radiographs, suggesting high sensitivity for the TOMS.

References:

[1] Kroon FPB, Conaghan P, Foltz V, et al. Development and reliability of the OMERACT thumb base osteoarthritis MRI scoring system. J Rheumatol. 2017; in press.

Disclosure of Interest: None declared

DOI: 10.1136/annrheumdis-2017-eular.2765

\section{SATURDAY, 17 JUNE 2017 Why we do develop autoimmunity}

\section{OP0343 THE INTESTINAL INVOLVEMENT IN SYSTEMIC SCLEROSIS IS CHARACTERIZED BY A PECULIAR GUT MICROBIOTA}

G. Natalello ${ }^{1}$, S.L. Bosello ${ }^{1}$, F. Paroni Sterbini ${ }^{2}$, A. Palladini ${ }^{3,4}$,

G.B. Canestrari ${ }^{1}$, F. Parisi ${ }^{1}$, E. De Lorenzis ${ }^{1}$, G. Berardi ${ }^{1}$, B. Posteraro ${ }^{5}$,

M. Sanguinetti ${ }^{2}$, G. Ferraccioli ${ }^{1}$. ${ }^{1}$ Institute of Rheumatology and Affine Sciences; ${ }^{2}$ Institute of Microbiology, Università Cattolica del Sacro Cuore, Rome, Italy; ${ }^{3}$ Paul Langerhans Institut Dresden of the Helmholtz Centre Munich at the University Clinic Carl Gustav Carus, TU Dresden, Dresden; ${ }^{4}$ German Center for Diabetes Research (DZD e.V.), Neuherberg, Germany; ${ }^{5}$ Institute of Public Health, Università Cattolica del Sacro Cuore, Rome, Italy

Background: Gastrointestinal involvement is recognized as a major cause of morbidity and mortality in Systemic Sclerosis (SSc) and its pathophysiology is still unclear. Few data on composition and function of gut microenvironment in SSc are reported in the literature but there is a growing body of evidences supporting the hypothesis of a relation between gut microbiota and the host immune system. Objectives: The goal of this study was to characterize fecal microbiota in SSc patients compared to healthy subjects to investigate whether specific microbial species may be responsible of dysbiosis in SSc. Furthermore, we investigated the composition of microbiota in the different clinical subsets of SSc.

Methods: Faecal samples were obtained from 17 healthy controls and 39 SSc patients including subjects with different skin involvement (Diffuse and Limited) and disease duration. The BMI was normal and the mean age was similar both in SSc and controls groups. The composition of microbiota was determined through $16 \mathrm{~S}$ rRNA pyrosequencing performed using the GS Titanium technology. Rarefaction was used to uniform abudance data. $\alpha$-diversity was defined by the main indexes while $\beta$-diversity was determined according to Bray-Curtis and UniFrac, represented trough Principal Coordinate Analysis (PCoA) and compared using PERMANOVA test on distance matrices. Linear Discriminant Analysis Effect Size was used to identify taxa that showed differential expression between the groups. Results: At genus level SSc patients showed a differential expression in 12 taxa compared to controls with higher levels of Ruminococcus, Streptococcus, 